\title{
Literacia em Saúde nos Doentes Hospitalizados num Serviço de Medicina Interna
}

\section{Health Literacy in Patients Hospitalized in an Internal Medicine Ward}

\begin{abstract}
Adriana Watts Soares (https://orcid.org/0000-0002-6166-3145), Maria Maia (https://orcid.org/0000-0001-5194-6865), Virginia Visconti (https://orcid.org/0000-0001-5815-0528), André Fernandes (https://orcid.org/0000-0002-3770-9510), João Espírito Santo (https://orcid.org/0000-0001-6415-2095), Isménia Oliveira1 (https://orcid.org/0000-0002-9207-7851), Francisco Araújo (https://orcid.org/0000-0002-6224-4866), José Lomelino Araújo (https://orcid.org/0000-0001-6855-2993)
\end{abstract}

\section{Resumo:}

Introdução: Literacia em saúde é um tema de popularidade crescente face à relevância e impacto que tem nos resultados clínicos. Existem diversas ferramentas de avaliação de literacia em saúde sendo o European Health Literacy Survey Questionnaire (HLS-EU) o mais recentemente aplicado e validado. Graus de literacia consideradas problemáticas ou insuficientes estão associadas a incidência e gravidade pior de doenças, taxas de hospitalização e morbi-mortalidade superior. O objetivo foi aferir o grau de literacia em saúde relativamente aos cuidados de saúde.

Material e Métodos: Aplicação da versão portuguesa do questionário HLS-EU (HLS-PT) aos doentes internados ao cuidado da medicina interna. Incluídos todos os doentes internados ao cuidado da medicina interna, tendo-se excluído de doentes cognitivamente e clinicamente incapazes de responder ao questionário.

Resultados: Foram inquiridos 64 doentes. A amostra era maioritariamente do sexo masculino (57\%) com média de idade 71 anos, reformado $(71,8 \%)$ e com grau de escolaridade baixa (56,2\% com formação académica até $1^{\circ}$ ciclo). A maioria $(68,7 \%)$ tinha grau de literacia em saúde problemática ou insuficiente quanto aos cuidados de saúde.

Conclusão: Apesar de se reconhecer que, tanto o ambiente da aplicação do questionário como as características demográficas deste estudo serem diferentes do estudo nacional e europeu, revela-se um resultado preocupante da literacia em saúde de indivíduos hospitalizados com doenças crónicas.

Palavras-chave: Comunicação; Hospitalização; Literacia em Saúde.

\section{Abstract:}

Introduction: Health literacy is a topic of growing popularity given its relevance and impact on clinical outcomes. There are several health literacy assessment tools. The European Health Literacy Survey Questionnaire (HLS-EU-Q) is the most recently validated in Europe, it has a validated Portuguese version and it

Serviço Medicina Interna, Hospital Beatriz Ângelo, Loures, Portugal https://revista.spmi.pt - DOI: 10.24950/O/233/19/2/2020 categorizes 4 different degrees of health literacy (excellent, sufficient, problematic and insufficient). Lower degrees of literacy are associated with worse incidence and severity of illness, hospitalization rates and higher morbidity and mortality. The goal was to assess the health literacy level in relation to healthcare.

Material and Methods: Application of the Portuguese version of the HLS-EU (HLS-PT) questionnaire to patients admitted to internal medicine care. All patients admitted to the internal medicine ward were initially included. Exclusion criteria were evidence of cognitive or clinical inability to answer the questionnaire.

Results: 64 patients were surveyed. Study population was mostly male (57\%) with an average age of 71 years, retired (71.8\%) and with low level of education (56.2\% with academic education up to junior school). The majority (68.7\%) had a problematic or insufficient health literacy.

Conclusion: Although the authors recognize that both the environment of the questionnaire application and the demographic characteristics of this study differ from the national and European study, it is a disturbing result regarding health literacy in hospitalized individuals with chronic diseases.

Keywords: Communication; Health Literacy; Hospitalization.

O tema de literacia em saúde tem vindo a ganhar importância quando se aborda estratégias de saúde pública, sendo considerado como um dos objectivos prioritários com planos de acção específicos por parte da Organização Mundial da Saúde e Direcção Geral da Saúde (DGS).

O conceito de literacia em saúde foi primeiramente definido em 1998 mas foi sofrendo alterações ao longo dos anos. Foi o grupo liderado por Kickbusch que, em 2005, publicou o artigo onde define literacia em saúde como "a capacidade para tomar decisões fundamentadas na utilização de serviços de saúde no mercado e no contexto político no decurso da vida do dia-a-dia, em casa, na comunidade, local e trabalho". ${ }^{1}$ Em 2011, o grupo liderado por Sorensen divulgou uma definição semelhante à de Kickbusch mas com maior ênfase na capacitação e responsabilização dos cidadãos para tomar decisões fundamentadas. ${ }^{2}$ 
Um grau de literacia em saúde menor condiciona a compreensão dos cuidados propostos na prevenção de doenças, na prestação dos cuidados, nos resultados que se pretende obter bem como na utilização eficaz dos sistemas de saúde nomeadamente na procura de medidas de promoção de saúde. Este grupo tem uma maior taxa de doenças crónicas, maior gravidade das mesmas e morbi-mortalidade, levando a uma taxa superior de hospitalização.

Estipula-se que o grau de literacia em saúde seja um factor preditor e definidor da desigualdade em saúde de diferentes grupos socioeconómicos, visto existir uma correlação positiva entre os dois. ${ }^{3-6}$ Apesar de não verificado em nenhum estudo, tem-se realçado o impacto económico que a literacia em saúde tem em gastos e custos em política de saúde. , $4,7^{3}$

Diversas ferramentas foram desenvolvidas para caracterizar o grau de literacia em saúde: umas dirigidas a determinadas doenças e outras destinadas a populações de diferentes ambientes socio económicos. ${ }^{2} \mathrm{O}$ instrumento aplicado e validado em 2011 em oito países europeus (Irlanda, Holanda, Alemanha, Áustria, Polónia, Bulgária, Grécia e Espanha) e posteriormente em Portugal em 2014 é o questionário intitulado European Health Literacy Survey Questionnaire (HLSEU-Q) que divide a literacia em saúde em três domínios, nomeadamente, prevenção de doença, promoção de saúde e cuidados de saúde (Tabela 1). 1,2,5 Avalia a percepção que os doentes têm relativamente à sua capacidade de aceder, compreender, criticar e aplicar a informação oferecida, classificando-as em "excelente", "suficiente", "problemática" e "inadequada" literacia em saúde. 1,2,5

Resumindo os resultados obtidos pela aplicação do HLSEU-Q europeu e inclusão de 8000 cidadãos, foi demonstrado que $47 \%$ dos participantes mostra níveis de literacia em saúde problemáticos ou inadequados, correlacionando-se este aspeto com idade avançada (> 75 anos), menor grau de escolaridade, dificuldades financeiras e existência de doenças crónicas. Em 2014, um grupo de investigadores da área de saúde pública aplicou a mesma ferramenta em Portugal, após tradução e validação nacional do questionário (HLS-EU-PT, anexo 1). ${ }^{5,6}$ Foram incluídos 1004 cidadãos acima de 16 anos de idade, de todas as regiões geográficas. Destaca-se que apenas $4,6 \%$ se encontravam numa faixa etária avançada (> 65 anos), 7,2\% eram reformados e que apenas 5,6\% tinha o $1^{\circ}$ ciclo de escolaridade. Os resultados mostraram níveis problemáticos ou inadequados em $55,8 \%$ relativamente a cuidados de saúde, valores esses que são superiores à média do estudo original europeu. Os dados obtidos com os diversos estudos nesta área, têm influenciado e motivado uma mudança de planos de acção por parte da DGS para otimizar os meios e o impacto da comunicação sobre literacia em saúde. ${ }^{7-11}$

\section{Os objetivos deste estudo são:}

1) caracterizar o grau de literacia em saúde relativamente ao domínio "cuidados de saúde" dos doentes internados ao cuidado da Medicina Interna e

2) conhecer as sugestões dos doentes para melhorar a literacia em saúde.

\section{Material e Métodos}

Trata-se de um estudo observacional, descritivo-exploratório, de abordagem quantitativa, com colheita das respostas ao inquérito HLS-EU-PT - Cuidados em Saúde (Tabela 2) a todos os doentes convidados a participar neste estudo internados ao cuidado da medicina interna em três enfermarias de um hospital terciário, em dois dias com três semanas de intervalo. Os inquéritos abaixo descritos foram preenchidos com colaboração dos investigadores, permitindo explicações sobre cada pergunta de modo a melhor compreensão e resposta mais fiável.

O estudo foi aprovado pela Comissão de Ética do hospital

Tabela 1: Matriz Traduzida do HLS-EU

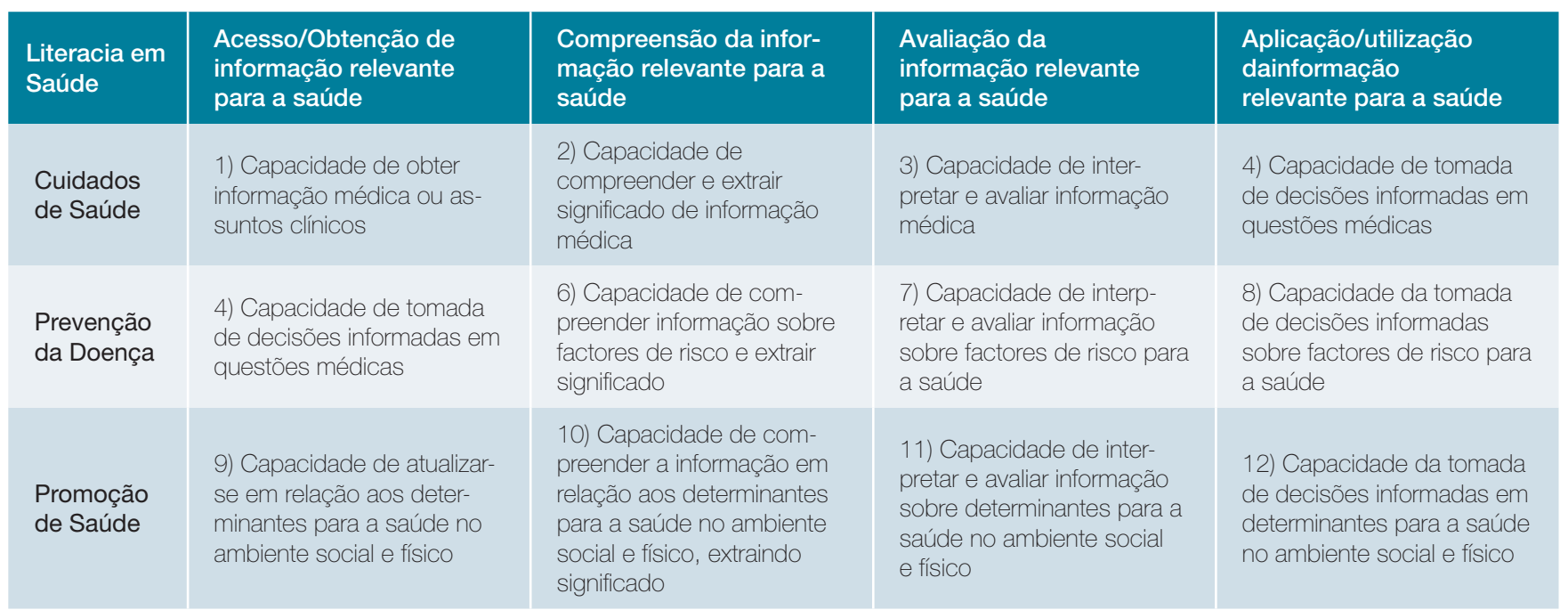


Tabela 2: Inquérito adaptado da versão portuguesa do Health Literacy Survey (HLS EU-PT) - Cuidados em Saúde

\begin{tabular}{|c|}
\hline HLS EU-PT - Cuidados em Saúde \\
\hline Numa escala de 1- Muito Fácil, 2- Fácil, 3- Difícil, 4- Muito Difícil, \\
5- Não Sabe Responder Quão difícil diria que:
\end{tabular}

Q 1. "...em encontrar informações sobre sintomas de doenças que Ihe dizem respeito ou preocupam?"

Q 2. "...em encontrar informações sobre tratamentos de doenças que the dizem respeito ou preocupam?"

Q 3. “... descobrir o que fazer em caso de emergência médica”

Q 4. "... descobrir onde obter ajuda especializada quando está doente? (por ex. junto de um médico, farmacêutico, psicólogo)

\section{Q 5. "...compreender o que o seu médico lhe diz?"}

Q 6. "...compreender a bula (os folhetos) que acompanham o seu medicamento?"

Q 7. "...compreender o que fazer numa emergência médica?"

Q 8. “...compreender instruções do seu médico ou farmacêutico sobre o modo de tomar um medicamento que the foi receitado?"

Q 9. "... avaliar como é que a informação proveniente do seu médico se aplica ao seu caso?"

Q 10. “... avaliar vantagens e desvantagens de diferentes opções de tratamento?"

Q 11. "... avaliar quando pode necessitar de uma segunda opinião de outro médico?"

Q 12. “... avaliar, se a informação sobre a doença, nos meios de comunicação, é de confiança?" (por ex. TV, Internet ou outros meios de comunicação)

Q 13. “... usar informações que o seu médico lhe dá para tomar decisões sobre a sua doença"

Q 14. "...seguir/cumprir instruções sobre medicação?"

Q 15. "...seguir/cumprir instruções sobre medicação?"

Q 16. “...seguir/cumprir as instruções do seu médico/ farmacêutico?"

Questionário Literacia em Saúde - Cuidados em Saúde

onde o estudo foi realizado.

Antes de convidar doentes a participar no estudo, foram feitas perguntas por dois examinadores, para aferir o grau de orientação auto e alopsíquica, temporal e espacial, de modo a evitar inclusão de doentes que não se encontravam num estado cognitivo apto para reconhecer participação no estudo ou compreender as questões colocadas, seja pelo estado cognitivo basal ou por estado confusional agudo decorrente do motivo de internamento.

Foi efetuada a caracterização demográfica (género, idade, escolaridade, situação laboral, estado civil), caracterização de capacidade cognitiva e grau de autonomia para atividades instrumentais utilizando inquéritos de autoavaliação a escala Clinical Dementia Rating-Sum of Boxes (CDR-SOB) ${ }^{8}$ e a escala de Lawton-Brody, ${ }^{9}$ respetivamente.
A escala CDR-SOB avalia o grau de compromisso que o inquirido tem relativamente a seis domínios de função cognitiva e social com 0,5 pontos por cada parâmetro afetado (memória, orientação, juízo/resolução de problemas, atividades sociais, atividades domésticas e recreativas e cuidados pessoais). A pontuação é somada e padronizada em valores de 0 a 3 relativamente a declínio cognitivo $(0,5$ - sem ou suspeita de declínio, 1-declínio cognitivo ligeiro, 2-declínio cognitivo moderado, 3-declínio cognitivo grave). ${ }^{4} \mathrm{~A}$ escala de Lawton-Brody quantifica o grau de autonomia para atividades instrumentais diárias (cuidar da casa, lavar a roupa, preparar refeições, fazer compras, usar o telefone, usar transporte, usar dinheiro e compliance farmacológica), equivalendo a autonomia total para cada item 1 ponto e a incapacidade a 3 a 5 pontos (método de pontuação de Sequeira C.5). A pontuação varia entre 8 a 30 pontos sendo independência definida como 8 pontos, dependência ligeira 9-10 pontos, dependência moderada 11-19 pontos e dependência grave $>20$ pontos. ${ }^{5}$

Aplicou-se o inquérito HLS-EU-PT aos doentes selecionados. Este instrumento é composto por quarenta e sete questões que abrangem os três domínios da literacia em saúde - cuidados de saúde, promoção de saúde e prevenção da doença - e quatro níveis de processamento da informação - acesso, compreensão, avaliação e utilização. A pontuação (1-5 pontos para cada questão, com nível crescente da autopercepção da dificuldade executar cada uma delas) é somada e padronizada em diferentes escalas de literacia ("excelente", "suficiente", "problemático" e "inadequado"). ${ }^{3}$ Neste estudo, os doentes foram questionados somente no sector de aferição de literacia relativa a cuidados de saúde e procurou-se comparar com os dados obtidos na aplicação do inquérito a nível nacional.

Os dados foram processados utilizando Microsoft Office Excel ${ }^{\circledR}$.

Relativamente ao segundo objetivo, no final da aplicação do inquérito, os indivíduos foram incentivados a sugerir como aumentar a literacia em saúde da população em geral. As respostas eram recolhidas em texto livre após ter sido perguntado ao inquirido pela opinião e sugestão de como melhorar a literacia em saúde em Portugal.

\section{Resultados}

De um total de 192 doentes internados nas três enfermarias, 139 estavam ao cuidado de Medicina Interna, dos quais 64 foram excluídos por incapacidade de colaboração no estudo e 11 por recusarem participar. Assim, foram inquiridos 64 doentes para os resultados do estudo. Amostra: A média de idade foi de 71 anos (mínimo 31 e máximo 92 anos) com predomínio do sexo masculino (37 doentes, 57\%) sendo que estes eram, em média, mais jovens do que os do sexo feminino (69 vs 74 anos). Quanto ao estado civil, $57,8 \%$ encontravam-se casados, $21,8 \%$ viúvos e $17 \%$ em situação de divórcio ou solteiro. 
Tabela 3: Caracterização demográfica, cognitiva e avaliação de autonomia da amostra

\begin{tabular}{|c|c|c|c|}
\hline Característica da amostra & Feminino & Masculino & Total \\
\hline Idade (anos) Mínima | Máxima | Média & 52 | 92 | 74 & 31 | 90| 69 & 31 | 92 | 71 \\
\hline $\begin{array}{l}\text { Estado civil Casado | Solteiro/divorciado } \\
\text { |Viúvo }\end{array}$ & 12 | 5 |10 & $25|6| 4$ & $\begin{array}{c}37(57 \%)|11(17,1 \%)| \\
14(21,8 \%)\end{array}$ \\
\hline $\begin{array}{l}\text { Situação laboral Desempregado | Empregado } \\
\text { | Reformado }\end{array}$ & $2|7| 18$ & $2|7| 28$ & $\begin{array}{c}4(6,25 \%)|14(21,8 \%)| \\
46(71,8 \%)\end{array}$ \\
\hline $\begin{array}{l}\text { Escolaridade Não frequentou } \mid 1 .^{\circ} \text { ciclo } \mid 2 .^{\circ} \mathrm{e} \\
3 .^{\circ} \text { ciclo | Ensino secundário | Ensino superior }\end{array}$ & $6|15| 2|2| 2$ & $3|21| 4|6| 3$ & $\begin{array}{c}9(14 \%)|36(56,2 \%)| \\
6(9,3 \%)|8(12,5 \%)| \\
5(7,8 \%)\end{array}$ \\
\hline Cognição (escala CDR-SOB) $0,5|1| 2 \mid 3$ & $19|4| 4 \mid 0$ & $33|4| 0 \mid 0$ & $\begin{array}{c}52(81,2 \%)|8(12,5 \%)| \\
4(6,25 \%) \mid 0\end{array}$ \\
\hline $\begin{array}{l}\text { Autonomia (escala Lawton-Brody) } \\
\text { Independente | Dependência ligeira | } \\
\text { Dependência moderada | Dependência grave }\end{array}$ & $6|3| 11 \mid 7$ & $6|8| 17 \mid 6$ & $\begin{array}{c}12(18,7 \%)|11(17,1 \%)| \\
28(43,7 \%) \mid 13(20 \%)\end{array}$ \\
\hline
\end{tabular}

Caracterização da amostra

Relativamente à atividade profissional, a salientar que 71\% dos doentes estavam reformados. A grande maioria apresentava um grau de escolaridade baixa sendo que 14\% não tinha frequentado nenhum nível de escolaridade e 56\% tinha concluído apenas o ensino básico. Por oposição, 20\% tinha completado ensino secundário ou superior. Relativamente à capacidade cognitiva, a avaliação CDR-SOB revelou que a maioria dos doentes inquiridos ou não tinham declínio cognitivo ou apresentava apenas suspeita de tal, compreendendo $81,25 \%$ da amostra, seguido de 12,5\% com declínio ligeiro e 6,25\% moderado. Quanto à autonomia para atividades instrumentais diárias, utilizando a escala de Lawton-Brody, a maioria dos inquiridos (43\%) apresentava dependência moderada, estando os restantes equitativamente distribuídos pelos diferentes graus de dependência: autonomia total (18,75\%), dependência ligeira $(17,2 \%)$ e dependência grave (20\%) (Tabela 3).

\section{GRAU DE LITERACIA EM SAÚDE - CUIDADOS DE SAÚDE}

O inquérito, como acima descrito, procura aferir a auto-perceção que o individuo tem na facilidade de aceder, compreender, criticar e aplicar a informação oferecida. Neste domínio específico - Cuidados de Saúde, as questões assentam sobre a capacidade de encontrar informação sobre doenças ativas e tratamentos seja em ambiente de consulta de ambulatório seja em situações de emergência, compreender as decisões dos médicos, avaliar e criticas as decisões tomadas ou pertinência de uma segunda opinião, procurar esclarecimento sobre vantagens e desvantagens de tratamentos ou efeito dos medicamentos, compreender e aplicar as instruções dos profissionais de saúde. Verificou-se que $68,75 \%$ da amostra apresenta grau de literacia problemática ou inadequada (Tabela 4).

Discriminando os níveis de processamento da informação, os inquiridos confessaram ter maior dificuldade para avaliar/ criticar a informação que Ihes foi dada pelo profissional de saúde (ver perguntas Q9-12 do anexo) comparativamente com compreensão e execução das instruções fornecidas $(Q$ 5-8 e Q 13-16). Reforçando este aspeto, apesar de não ter significância estatística, a moda e a média da pontuação dada às questões no âmbito de avaliação da informação foi de 3 e 2,8, respectivamente, ao invés de uma moda de 2 e uma

Tabela 4: Resultados. Justaposição dos dados obtidos

\begin{tabular}{|c|c|c|c|c|}
\hline Grau de literacia & Excelente & Suficiente & Problemático & Inadequado \\
\hline Parâmetro & $6 / 64$ inquiridos & 14/64 inquiridos & 23/64 inquiridos & $21 / 64$ inquiridos \\
\hline Idade, anos (média) & 65 & 67 & 72 & 75 \\
\hline $\begin{array}{l}\text { Capacidade cognitiva Escala } \\
\text { CDR-SOB (pontos, média) }\end{array}$ & 0,5 & 0,5 & 0,6 & 0,8 \\
\hline $\begin{array}{l}\text { Capacidade autonomia Escala } \\
\text { Lawton-Brody (pontos, média) }\end{array}$ & 8,5 & 10,7 & 16,1 & 16,9 \\
\hline $\begin{array}{l}\text { Escolaridade Não frequentou } \\
\mid 1 .^{\circ} \text { ciclo } \mid 2 .^{\circ} \text { e } 3 .^{\circ} \text { ciclo } \mid \\
\text { Ensino secundário | Ensino } \\
\text { superior }\end{array}$ & $0|3| 0|2| 1$ & $0|9| 0|4| 1$ & $3|12| 4|1| 3$ & $6|12| 3|0| 0$ \\
\hline
\end{tabular}




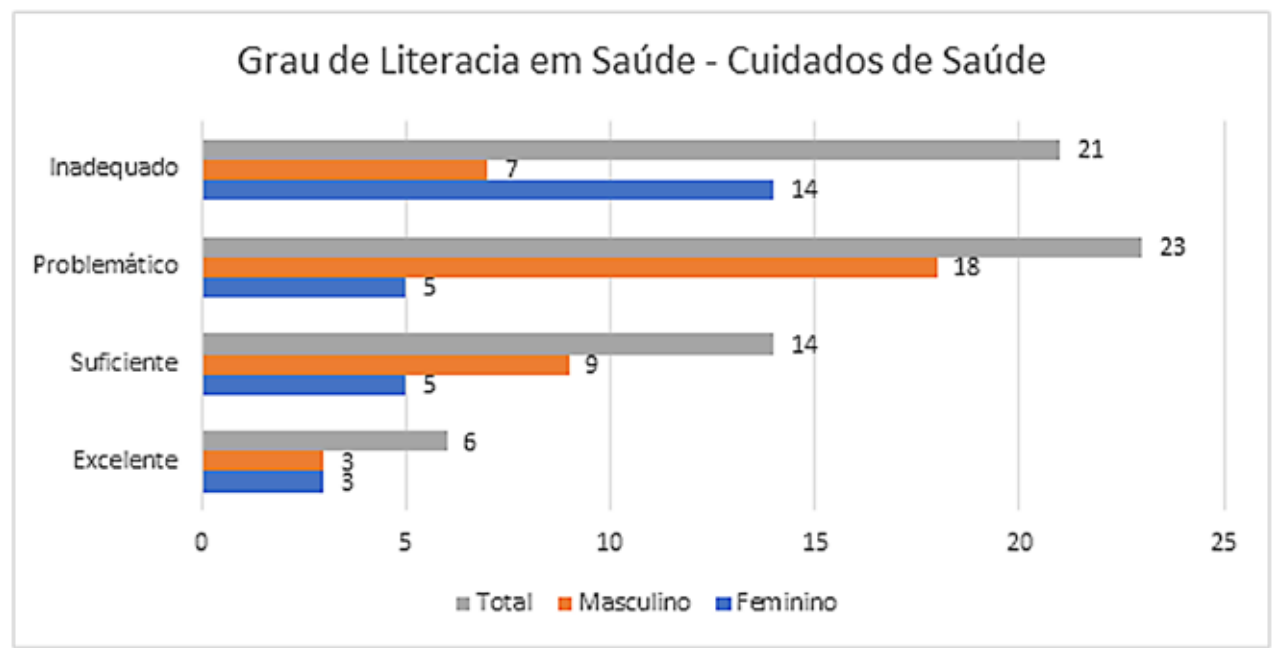

Figura 1: Resultados. Grau de Literacia em Saúde - Cuidados de Saúde dos doentes inquiridos.

média inferior a 2,5 nos restantes domínios de processamento da informação (Fig. 1).

\section{SUGESTÃO DOS DOENTES PARA AUMENTAR LITERA- CIA EM SAÚDE}

Muitos doentes mostraram-se inseguros para opinar. Daqueles doentes que quiseram partilhar uma sugestão, a maioria sugeriu uma das seguintes opções:

1) aumentar o acesso, a facilidade e o tempo de consulta com os médicos, citando que os médicos de cuidados primários eram os mais adequados para esclarecer à população sobre a saúde e doença;

2) que os médicos utilizassem uma linguagem mais adequada à população.

O doente mais idoso deste grupo sugeriu criar uma linha telefónica no centro de saúde para atendimento telefónico por enfermeiros ou médicos com horário específico para tal, onde os cidadãos colocariam questões que surgissem no seu diaa-dia relativamente a saúde ou doença, com impacto direto na sua vida. Outros dois indivíduos sugeriram uma adaptação das soluções já existentes de modo a atingir uma população mais jovem. Uma destas sugestões abordava a comunicação sobre saúde em tele-jornalismo, devendo priorizar os horários noturnos. Falaram de saber da existência de programas de televisão ou telejornais onde médicos são convidados para falar sobre temas no âmbito da saúde de interesse para a literacia em saúde, mas que tendem a ser horários matinais, alturas do dia em que o horário laboral impede a visualização destes programas. A outra sugestão foi de facilitar o acesso a manuais/artigos informativos escritos pela DGS à população.

\section{Discussão}

Os resultados deste estudo mostraram que o grau de literacia em saúde da amostra inquirida é muito baixo, tal como os resultados do estudo da aplicação da mesma ferramenta a nível nacional. Reconhece-se que as amostras dos estudos eram diferentes, tanto nas características demográficas (população mais idosa, nível de escolaridade inferior) como no ambiente em que o inquérito foi aplicado (hospital versus na comunidade). Contudo, fazendo uma comparação entre os resultados obtidos pelo estudo nacional em 2014, a nossa amostra revela uma pontuação mais preocupante $(68,75 \%$ com literacia problemática ou inadequada vs 55,8\%). Propomos como fatores explicativos desta discrepância, pelo menos parcialmente, a localização geográfica e tipo de ambiente (urbano, suburbano e rural) de acordo com aspetos culturais e investimento local na educação. Quando feita a justaposição de todos os dados obtidos da nossa amostra, parece existir uma relação positiva entre os índices de capacidade cognitiva e autonomia e uma negativa entre idade com a literacia em saúde independentemente do grau de escolaridade.

Relativamente às sugestões dos doentes, estes realçaram a necessidade de maior acessibilidade aos profissionais de saúde (médicos de cuidados primários) como a utilização de uma linguagem mais compreensível para a população. Estas respostas parecem ser algo contraditórias e refletem alguns aspetos interessantes. Ao mesmo tempo que os doentes atribuem somente aos médicos a responsabilidade de educar sobre a saúde, criticam que a comunicação utilizada é insatisfatória para os fins a que se propõe. Possivelmente por preconceito, desenvolvido por questões históricas e hábitos culturais, não apontam a classe de enfermagem como um veículo de formação em saúde, algo que os autores acreditam que podia ter um impacto favorável pois aumentaria o número de participantes no plano de ação em prol da literacia em saúde. Caracterizam, também, o volume e o tempo de consulta como a plataforma de ensino sobre saúde/doença, considerando a aprendizagem ser fruto de uma relação médico-doente individualizada.

Não obstante, uma amostra muito pequena revelou compreender a diferença entre esclarecimento pessoal de dúvidas sobre saúde e doença e literacia em saúde da população. 
Destes doentes, surgiram sugestões interessantes. Alguns inquiridos sugeriram uma utilização mais eficaz dos meios de comunicação já existentes, por exemplo - melhor aproveitamento dos tele-jornais para abordar temas de saúde relevantes, devendo priorizar os horários noturnos. Sugeriram também edição da plataforma online da DGS para facilitar a navegabilidade na mesma para extrair a informação que se procura (seja sobre temas de saúde como horários de acesso a cuidados primários para doença aguda, etc.). A plataforma online da DGS é rica em informação, tendo revistas digitais para consulta sobre assuntos de interesse na área de promoção de saúde, explicação de como utilizar os recursos e navegar dentro do sistema nacional de saúde; exposição das iniciativas comunitárias para diversas ações para consciencialização para hábitos de vida mais saudáveis; explicação sobre hábitos alimentares, etc. No entanto, esta plataforma online é pouco divulgada nos meios de comunicação; não aparece, automaticamente, no topo do motor de pesquisas quando se introduzem palavras-chave que mostram intenção de pesquisar sobre temas de saúde ou doença, perdendo lugar para "blogs" pessoais e de opinião. Portanto, depreende-se que as sugestões pedem um ajuste ao que já existe de forma a que a população com grau de escolaridade mais avançado, com trabalho ativo se sinta abrangida nos programas de ação para a literacia em saúde.

\section{Limitações}

Reconhece-se que a amostra, para além de ser diferente daquela inquirida no estudo nacional e europeu, é relativamente pequena para abordar um assunto com um impacto tão importante em saúde pública, não permitindo fazer ilações significativas nem alavancar ações de melhoria. Contudo, os resultados obtidos decretam estimular uma reflexão sobre a literacia em saúde na população que servimos. Teria sido interessante aferir o grau de literacia em saúde dos cuidadores dos doentes que foram excluídos da amostra, dado ser igualmente pertinente que os mesmos saibam aceder à informação acerca de saúde/doença e compreender a sua aplicação para benefício dos indivíduos deles dependentes. Por fim, é sabido que o internamento por si só se associa tanto a angústia como a delirium levando a alterações da cognição e capacidade de orientação do doente, facto que pode contribuir para a subvalorização do nível de literacia em saúde. É criticável não se ter utilizado ferramentas de diagnóstico de delirium objetivos. No entanto, como descrito nos métodos, os doentes foram submetidos a perguntas para aferir existência de estado confusional que revelasse inaptidão de reconhecer participação no estudo ou compreender as questões colocadas.

\section{Conclusão}

Existe um muito baixo grau de literacia em saúde, nesta pequena amostra de doentes internados ao cuidado da
Medicina Interna. Analisando os diferentes parâmetros demográficos dos doentes inquiridos, parece existir uma relação positiva entre o grau de literacia em saúde com os índices de capacidade cognitiva e autonomia. Por outro lado, tal como os estudos europeu e nacional refletem, a idade tem um impacto negativo na literacia em saúde, independentemente do grau de escolaridade do doente. Atendendo ao objetivo de melhorar o grau de literacia em saúde em Portugal, são visíveis os resultados por parte do departamento da DGS responsável por este assunto com a criação de websites destinados a difundir informação sobre promoção e cuidados de saúde e navegação no sistema nacional de saúde com uma linguagem mais próxima da população. No entanto, perguntase se estas plataformas são suficientemente divulgadas para a classe etária que utiliza a internet como fonte de conhecimento e se é acessível aos utentes mais idosos. Levanta-se também a questão se poderia haver uma colaboração mais eficaz entre a comunicação televisa/jornalística com a DGS para abranger uma população maior de modo a promover a literacia em saúde mais amplamente.

\section{Agradecimentos}

Agradeço a colaboração e empenho dos alunos Marta Machado, Rodrigo Varandas, João Silva, Madalena Machete e Raquel Branco, que permitiram executar este trabalho.

\section{Responsabilidades Éticas}

Conflitos de Interesse: Os autores declaram a inexistência de conflitos de interesse na realização do presente trabalho.

Fontes de Financiamento: Não existiram fontes externas de financiamento para a realização deste artigo.

Confidencialidade dos Dados: Os autores declaram ter seguido os protocolos da sua instituição acerca da publicação dos dados de doentes. Proteção de Pessoas e Animais: Os autores declaram que os procedimentos seguidos estavam de acordo com os regulamentos estabelecidos pelos responsáveis da Comissão de Investigação Clínica e Ética e de acordo com a Declaração de Helsínquia da Associação Médica Mundial. Proveniência e Revisão por Pares: Não comissionado; revisão externa por pares.

\section{Ethical Disclosures}

Conflicts of interest: The authors have no conflicts of interest to declare. Financing Support: This work has not received any contribution, grant or scholarship

Confidentiality of Data: The authors declare that they have followed the protocols of their work center on the publication of data from patients.

Protection of Human and Animal Subjects: The authors declare that the procedures followed were in accordance with the regulations of the relevant clinical research ethics committee and with those of the Code of Ethics of the World Medical Association (Declaration of Helsinki).

Provenance and Peer Review: Not commissioned; externally peer reviewed. 
(C) Autor (es) (ou seu (s) empregador (es)) 2019. Reutilização permitida de acordo com CC BY-NC. Nenhuma reutilização comercial.

(C) Author(s) (or their employer(s)) 2019. Re-use permitted under CC BY-

NC. No commercial re-use.

\section{Correspondence / Correspondência:}

Adriana Watts Soares - adrianawsoares@gmail.com

Serviço Medicina Interna, Hospital Beatriz Ângelo, Loures, Portugal

Av. Carlos Teixeira 3, 2674-514 Loures

Received / Recebido: 20/11/2019

Accepted / Aceite: 03/02/2020

Publicado / Published: 27 de Junho de 2020

\section{REFERÊNCIAS}

1. Kickbusch I. Navigating Health: The Role Of Health Literacy. London: Alliance For Health And The Future, International Longevity Centre; 2005.

2. Sørensen K, Van den Broucke S, Pelikan JM, Fullam J, Doyle G, Slonska $Z$, et al. Measuring Health Literacy In Populations: Illuminating The Design And Development Process Of The European Health Literacy Survey Questionnaire (HIs-Eu-Q). BMC Public Health. 2013;13:948. doi:
10.1186/1471-2458-13-948

3. Pedro AR, Amaral O, Escoval A. Literacia Em Saúde, Dos Dados À Ação: Tradução, Validação E Aplicação Do European Health Literacy Survey Em Portugal. Rev Port Saúde Pública. 2016;34:259-75.

4. Literacia Em Saúde Em Portugal Relatório Síntese. Lisboa: Fundação Calouste Gulbenkian; 2016.

5. Saboga-Nunes L. Cross-Cultural Adaptation And Validation To Portuguese Of The European Health Literacy Survey (HLS-EU-PT). Aten Primaria. 2014, 46: 13

6. Marques P. Literacia Em Saúde: Avaliação Através do European Health Literacy Survey Em Português Num Serviço De Internamento Hospitalar [Tese de Mestrado]. Lisboa: ISCTE-Business School; 2015.

7. Espanha R, Avila P. Health Literacy Survey Portugal: a Contribution for the Knowledge on Health and Communications. Procedia Comp Sci.2016; 100: $1033-41$.

8. O'Bryant SE, Waring SC, Cullum CM, Hall J, Lacritz L, Massman PJ, et al. Staging Dementia Using Clinical Dementia Rating Scale Sum of Boxes Scores. Arch Neurol. 2008; 65: 1091-5. doi: 10.1001/archneur.65.8.1091.

9. Sequeira C. Cuidar de idosos dependentes. Coimbra: Quarteto Editora; 2007.

10. Despacho n. ${ }^{\circ} 3618-A / 2016$, Diário Da República, 2. a Série N. 4910 De Março De 2016, 8660-(5)-(6)

11. Plano De Ação, Literacia Em Saúde -Portugal 2019-2021, Direção de Serviços de Prevenção da Doença e Promoção da Saúde (DSPDPS) Divisão de Literacia, Saúde e Bem-Estar, Direção-Geral da Saúde, 2019. Lisboa: DGS; 2019. 